\title{
Adaptive Control of Switched Reluctance Motor Drives under Variable Torque Applications
}

\author{
Mohammad Masoud Namazi ${ }^{\dagger}$, Amir Rashidi*, Hamidreza Koofigar**, \\ Seyed Morteza Saghaiannejad* and Jin-Woo Ahn***
}

\begin{abstract}
This paper presents an adaptive control strategy for the speed control of a four-phase switched reluctance motor (SRM) in automotive applications. The main objective is to minimize the torque ripples, despite the unstructured uncertainties, time-varying parameters and external load disturbances. The bound of perturbations is not required to be known in the developing of the proposed adaptive-based control method. In order to achieve a smooth control effort, some properties are incorporated and the proposed control algorithm is constructed using the Lyapunov theorem where the closed-loop stability and robust tracking are ensured. The effectiveness of the proposed controller in rejecting high perturbed load torque with smooth control effort is verified with comparing of an adaptive sliding mode control (ASMC) and validated with experimental results.
\end{abstract}

Keywords: Switched reluctance motor, Ripple minimization, Adaptive control, Automotive applications

\section{Introduction}

During the last decade, various investigations have been devoted to the field of switched reluctance motors due to such advantages as lower costs and higher reliability, over PM-based machines in automotive applications [1]. Moreover, SRM is becoming increasingly a candidate for Electric Vehicles (EVs) due to its extended speed range at constant power that can minimize the power rating of the propulsion system for high acceleration performance and maximize the capability of recovering energy during regenerative braking operation [2]. However, its high torque ripple overwhelmed these advantages, especially for the usage as a traction motor in EVs with high driving torque that vibration force directly applied on wheels $[3,4]$. Therefore, advanced control methods are needed to reduce the torque ripple to deliver smoother power to the wheels $[5,6]$. This can be achieved by applying smoother control effort even in the presence of time-varying torque perturbations to reduce mechanical vibration and enhance the comfort level for the occupants $[7,8]$.

On the other hand, double saliency structure, inherent magnetic saturation and time variation of parameters form an uncertain nonlinear mathematical model for the SRM. Removing some drawbacks of the conventional mechanical

$\dagger \quad$ Corresponding Author: Dept. of Electrical and Computer Engineering, Isfahan University of Technology, Iran. (mm.namazi@ec.iut.ac.ir)

* Dept. of Electrical and Computer Engineering, Isfahan University of Technology, Iran. (\{a.rashidi, saghaian\}@cc.iut.ac.ir)

** Dept. of Electrical Engineering, University of Isfahan, Iran. (koofigar@eng.ui.ac.ir)

*** Dept. of Mechatronics Engineering, Kyungsung University, Korea. (jwahn@ks.ac.kr)

Received: May 4, 2016; Accepted: July 4, 2016 structure design, some advanced nonlinear control methods such as robust control strategies $[9,10]$ have been developed to achieve a minimized torque ripple performance. The torque sharing function (TSF), as one of the most significant used methods, may present a promising solution to minimize the torque ripple in SRM drives [11-14]. In [11], a study has been done on improving TSF for negative torque compensation in high speed applications. A novel family of optimal TSFs considering copper loss minimi-zation is proposed in [12]. However, working in positive torque producing region and using linear magnetic characterization may reduce the drive efficiency. The researchers have improved the TSF with the main objective of torque ripple minimization in wide speed range and two secondary objectives of minimization of power loss and rate of change of flux-linkage [13, 14]. In such works, the developed hysteresis current controller may have an effect on switching frequency of the power semiconductor devices when a lower hysteresis band is used. Moreover, the robustness analysis in the presence of unstructured uncertainties has not been given.

Higher order sliding mode controls, e.g., second order sliding mode (SOSM), have been also used to relax the chattering problem in the speed control of SRM [15]. However, the model uncertainties have not been considered due to difficulties in stability and robustness analysis. To address the above-mentioned issues, a novel integral sliding mode control method is proposed in [16] to achieve a faster torque response with acceptable ripple. Nevertheless, in such cases as automotive applications, the upper bound of perturbations may be unknown due to the uncertainty and time variation in load torque. The inadequacy of most controllers based on a sliding mode design lies in the 
treatment of such upper bound by calculating a switching gain. By increasing the gain for some large uncertainties, the high frequencies may be excited and some problems in hardware operation and chattering are inevitable. In general, chattering-free SMCs may be classified into those interacting with switching gain selection and those modifying the sliding function. Recently, a considerable attention has been paid to construct the combinational techniques to achieve a chattering-free performance via combining the SMC and gain adaptation techniques to develop adaptive sliding mode control for synchronous reluctance [17], and induction motor [18]. An adaptive sliding mode controller is designed in [19] which focused on the application of the linear switched reluctance actuator.

This paper proposes an adaptive control, as an approach for speed control of SRM with robust tracking performance under the unstructured uncertainties. The proposed scheme is capable of rejecting unknown time-varying load torque disturbance with attenuating the torque ripple. Moreover, the smoothness in the control effort provides a superior performance in speed tracking, compared with an adaptive SMC method, despite the both unstructured electrical and mechanical uncertainties of SRM. The validity and effectiveness of the proposed controller algorithm have been demonstrated by simulation and experiment. Experimental results are presented by implementing a drive system, including a $4 \mathrm{~kW}$, four phases $8 / 6 \mathrm{SRM}$. The results show the reasonable performance of the proposed strategy by comparing with ASMC.

\section{The Dynamic Model of SRM}

The SRM possesses a simple design with salient poles on both the rotor and stator but without windings on its rotor. Operation is based on the tendency to stand in alignment with the rotor and stator poles which yields a stable position and consequently, the reluctance is minimized. Torque production is due in sequential excitation of diametrically opposite stator poles by a switching algorithm [20]. The basic sets of electrical and mechanical differential equations are used for dynamic modeling of SRM can be stated as:

$$
\begin{gathered}
u_{\mathrm{j}}=r i_{\mathrm{j}}+\frac{\mathrm{d} \lambda_{\mathrm{j}}(\theta, i)}{\mathrm{d} t}, \\
J \dot{\omega}=T_{\mathrm{e}}(i, \theta)-T_{\mathrm{L}}-B \omega
\end{gathered}
$$

where $u$ is the voltage, $i$ denotes the current, $\lambda$ is the flux-linkage, $\mathrm{r}$ is the phase resistance, and $j=1,2,3,4$ stands for each phase of the machine. Moreover, $\mathrm{J}$ is the inertia of the motor, B denotes the motor friction, $T_{\mathrm{e}}(i, \theta)$ is the electromagnetic torque depends both on the angular rotor position and $T_{\mathrm{L}}$ shows the load torque.

\section{Adaptive Sliding Mode Control (ASMC) Design}

By taking the uncertainties into account just in the mechanical part of SRM dynamic model, this section focused on estimating the lumped mechanical uncertainties. Based on (1), the dynamic model of the SRM can be expressed as:

$$
\dot{\omega}=(a+\Delta \mathrm{a}) \omega+(b+\Delta b)\left(T_{e}(i, \theta)-T_{\mathrm{L}}\right)
$$

where $a=-\frac{B}{J}$ and $b=\frac{1}{J}$ denotes the nominal parameters and $\Delta a$ and $\Delta b$ are unknown parts. Defining the speed error $\mathrm{e}=\omega^{*}-\omega$, where $\omega^{*}$ is a reference speed, the sliding surface may be adopted as $S_{1}=\mathrm{c} e+\dot{e}, \mathrm{c}>0$ and its derivative is:

$$
\dot{S}_{1}=\mathrm{c} \dot{e}+\ddot{e}=-\mathrm{c} \dot{\omega}-\ddot{\omega}
$$

By substituting the derivative of $\dot{\omega}$ from (1) into (3), one obtains:

$$
\dot{S}_{1}=-\mathrm{c} \dot{\omega}-\left(\frac{B}{J} \dot{\omega}+\frac{1}{J}\left(\dot{T}_{e}-\dot{T}_{L}\right)\right)=-\mathrm{c} \dot{\omega}-\left(a \dot{\omega}+b\left(\dot{T}_{e}-\dot{T}_{L}\right)\right)
$$

Incorporating the defined uncertainties in (4), it can be represented as:

$$
\begin{aligned}
\dot{S}_{1} & =-\mathrm{c} \dot{\omega}-(a+\Delta a) \dot{\omega}-(b+\Delta b)\left(\dot{T}_{e}-\dot{T}_{L}\right) \\
& =-(\mathrm{c}+a) \dot{\omega}-b \dot{T}_{e}+b \dot{T}_{L}-b\left[\frac{1}{b}\left(\dot{\omega} \Delta a+\Delta b\left(\dot{T}_{e}-\dot{T}_{L}\right)\right)\right]
\end{aligned}
$$

By defining $P(t)=\frac{1}{b}\left(\dot{\omega} \Delta a+\Delta b\left(\dot{T}_{e}-\dot{T}_{L}\right)\right)$ as the lumped uncertainty, the sliding dynamic (5) can be rewritten as:

$$
\dot{S}_{1}=-(\mathrm{c}+a) \dot{\omega}-b \dot{T}_{e}+b \dot{T}_{L}-b P(t)
$$

Using the approximated inductance profile of the SRM, by neglecting magnetic saturation, the average torque $T_{\mathrm{e}}(i, \theta)$ can be expressed as [21]:

$$
T_{\mathrm{e}}=\frac{1}{2} i^{2} \frac{\mathrm{d} L(\theta)}{\mathrm{d} \theta}=\frac{1}{2} \frac{L_{a}-L_{u}}{\theta_{2}-\theta_{1}} i^{2}=\frac{1}{2} m i^{2}
$$

where, $L_{u}$ is the inductance at unaligned position $\theta_{1}, L_{a}$ is the inductance at aligned position $\theta_{2}$, and constant $m$ is the slope of the inductance corresponding to rotor position. Considering the phase current as a control input, one can obtain $\dot{T}_{\mathrm{e}} \propto i=u$ and the dynamic Eq. (6) can be written as: 


$$
\dot{S}_{1}=-(\mathrm{c}+a) \dot{\omega}-b u+b \dot{T}_{L}-b P(t)
$$

Now, define the Lyapunov function candidate:

$$
V_{1}=\frac{1}{2} S_{1}^{2}+\frac{1}{2 \rho} \tilde{P}^{2}(t)
$$

In which $\rho$ is a positive constant and $\tilde{P}(t)=$ $P(t)-\hat{P}(t)$ is the error between the actual value of the lumped uncertainty $P(t)$ and its estimated value $\hat{P}(t)$. The derivative of (9) is obtained as (the detailed manipulations are given in Appendix A):

$$
\begin{aligned}
\dot{V}_{1}= & -K_{1} S_{1}^{2}+S_{1}\left[-(\mathrm{c}+a) \dot{\omega}-b(u+\hat{P})+b \dot{T}_{\mathrm{L}}+K_{1} S_{1}\right] \\
& -\tilde{P}\left(\frac{1}{\rho} \dot{\hat{P}}+b S_{1}\right)
\end{aligned}
$$

Where $K_{1}$ a positive constant. Choose the control input $u$ as:

$$
u=\frac{1}{b}\left[-(\mathrm{c}+a) \dot{\omega}-b \hat{P}+b \dot{T}_{\mathrm{L}}+K_{1} S_{1}\right]
$$

Substitution the control law (11) in (10), one can obtain:

$$
\dot{V}_{1}=-K_{1} S_{1}^{2}-\tilde{P}\left(\frac{1}{\rho} \dot{\hat{P}}+b S_{1}\right)
$$

The adaptation law is defined as:

$$
\dot{\hat{P}}=-\rho b S_{1}
$$

Hence, adopting (13) in (12) gives:

$$
\dot{V}_{1}=-K_{1} S_{1}^{2} \leq 0
$$

where $\dot{V}_{1}$ is a negative semi-definite function and according to the Lyapunov stability theorem, the asymptotical stability of the control system is guaranteed, and the speed error asymptotically converges to zero [22].

\section{The Proposed Adaptive Control Design}

The previous section focused on using an adaptive scheme to estimate the lumped uncertainties. However, only the structured mechanical uncertainties are considered in the model of SRM. This section proposes an improved adaptive control scheme for eliminating this drawback by incorporating both mechanical and unstructured electrical uncertainties in the SRM model despite the time-varying load torque disturbances. The upper bound of perturbations is not required in the design procedure and is estimated by an adaptation mechanism to make the control gain small enough. This algorithm is also used the exponential functions as an effective solution to smoothen the control effort. The torque and current dynamics can be written from (1) as:

$$
\begin{gathered}
\frac{\mathrm{d} T_{\mathrm{e}}(i, \theta)}{\mathrm{d} t}=\frac{\partial T_{\mathrm{e}}(i, \theta)}{\partial i} \frac{\mathrm{d} i}{\mathrm{~d} t}+\frac{\partial T_{e}(i, \theta)}{\partial \theta} \frac{\mathrm{d} \theta}{\mathrm{d} t} \\
\frac{\mathrm{d} i}{\mathrm{~d} t}=\left(\frac{\partial \lambda(i, \theta)}{\partial i}\right)^{-1}\left(-r i-\frac{\partial \lambda(i, \theta)}{\partial \theta} \omega+\mathrm{u}\right)
\end{gathered}
$$

Both phase torque, $T_{\mathrm{e}}(i, \theta)$ and phase flux-linkage, $\lambda(i, \theta)$ are nonlinear functions of the phase current and the rotor position and for notational consistency, their arguments i.e., $i$ and $\theta$, are omitted in the following procedure. Substituting (16) in (15), (15) can be expressed as [21]:

$$
\frac{\mathrm{d} T_{e}}{\mathrm{~d} t}=\frac{\partial T_{\mathrm{e}}}{\partial i}\left(\frac{\partial \lambda}{\partial i}\right)^{-1}\left(-r i-\frac{\partial \lambda}{\partial \theta} \omega\right)+\frac{\partial T_{e}}{\partial \theta} \omega+\frac{\partial T_{\mathrm{e}}}{\partial i}\left(\frac{\partial \lambda}{\partial i}\right)^{-1} \mathrm{u}
$$

Therefore, the SRM dynamic model can be described in an affine form as [21]:

$$
\frac{\mathrm{d} T_{\mathrm{e}}}{\mathrm{d} t}=f\left(T_{\mathrm{e}}\right)+g\left(T_{\mathrm{e}}\right) u
$$

where the nonlinear functions $f\left(T_{\mathrm{e}}\right)$ and $g\left(T_{\mathrm{e}}\right)$ are specified by:

$$
\begin{gathered}
f\left(T_{\mathrm{e}}\right)=\frac{\partial T_{\mathrm{e}}}{\partial i}\left(\frac{\partial \lambda}{\partial i}\right)^{-1}\left(-r i-\frac{\partial \lambda}{\partial \theta} \omega\right)+\frac{\partial T_{e}}{\partial \theta} \omega, \\
g\left(T_{\mathrm{e}}\right)=\frac{\partial T_{\mathrm{e}}}{\partial i}\left(\frac{\partial \lambda}{\partial i}\right)^{-1}
\end{gathered}
$$

Defining $y=\omega$ as the output, and neglecting $B$, the dynamic model can be represented as:

$$
J \ddot{\omega}=f\left(T_{\mathrm{e}}\right)+g\left(T_{\mathrm{e}}\right) u-\mathrm{T}_{\mathrm{u}}(t)
$$

where $\mathrm{T}_{\mathrm{u}}(t)=\dot{T}_{\mathrm{L}}(t)$. As a preliminary step, rewrite (21) in the second-order compact affine form as:

$$
D \ddot{\omega}+\mathbf{C Q}=u-J^{-1} D \mathrm{~T}_{\mathrm{u}}(t)
$$

where:

$$
D=J \frac{\partial \lambda}{\partial i}\left(\frac{\partial T_{\mathrm{e}}}{\partial i}\right)^{-1}, \mathbf{C}=\left[\frac{\partial \lambda}{\partial \theta}-\frac{\partial T_{e}}{\partial \theta} \frac{\partial \lambda}{\partial i}\left(\frac{\partial T_{\mathrm{e}}}{\partial i}\right)^{-1} r\right]^{T}
$$


$\mathbf{Q}=\left[\begin{array}{ll}\omega & i\end{array}\right]$

Assumption 1. The uncertain nonlinear term $D$ is decomposed as $D=D_{0}+\Delta D$ where $D_{0}$ is the known part and $\Delta D$ represents the bounded unmodeled perturbation with bounded time-derivative, i.e., $|\Delta D|<z_{1}$ and $\left|\frac{\mathrm{d} \Delta D}{\mathrm{dt}}\right|<z_{2}$ where $z_{1}$ and $z_{2}$ are unknown constants and $z=\min \left\{z_{1}, z_{2}\right\}$.

Assumption 2. The parameters $\frac{\partial T_{e}}{\partial \theta}, \frac{\partial \lambda}{\partial i}, \frac{\partial T_{\mathrm{e}}}{\partial i}$ and $\frac{\partial \lambda}{\partial \theta}$ are time-varying with unknown bounds. This partial derivatives of flux and torque with respect to current and position are calculated by using the electromagnetic characteristics, achieved by the finite element method and verified by experimental measurement. The applied external torque $\mathrm{T}_{\mathrm{u}}(t)$ denotes the rate of variations in applied torque, assumed here as a time-varying disturbance with unknown bound value.

Assumption 3. By augmenting the unknown uncertainty $\mathbf{C}$, and the external load disturbance $\mathrm{T}_{\mathrm{u}}(t)$, augmented unknown time-varying vector and regressor vector are defined as:

$$
\begin{gathered}
\xi(t)=\left[\begin{array}{ll}
\mathbf{C} & T_{u}(t)
\end{array}\right]^{T} \\
\Psi=\left[\begin{array}{ll}
\mathbf{Q} & J^{-1} D
\end{array}\right]
\end{gathered}
$$

The unknown vector $\xi(t)$ is norm-bounded with an unknown value i.e., $\|\xi(\mathrm{t})\|<\mathrm{w}$ where $\mathrm{w}>0$ is an uncertain parameter.

Remark 1. By Assumptions 1-3, all kinds of SRM model uncertainties and disturbances, are taken into account, without any pre-assumption on the bound of such perturbations.

Remark 2. The term $g\left(T_{\mathrm{e}}\right)$ is not zero to derive the control law. This assumption is always satisfied for SRM, as the expressions $\frac{\partial \lambda}{\partial i}$ and $\frac{\partial T_{\mathrm{e}}}{\partial i}$ are shown to be positive. Considering $\lambda=\mathrm{L}(\theta, i) i$ one obtains:

$$
\frac{\partial \lambda(\theta, i)}{\partial i}=\frac{\partial(\mathrm{L}(\theta, i) i)}{\partial i}=L(\theta, i)+i \frac{\partial L(\theta, i)}{\partial i}
$$

From a physical viewpoint, it is true to assume the positivity of (25) in the range of operation [9], i.e.:

$$
L(\theta, i)+i \frac{\partial L(\theta, i)}{\partial i}>\mu>0
$$

Moreover, consider the fact that the rotor pole arc of the used SRM is larger than its stator pole arc, and the inductance profile does not contain the flatness characteristic [23]. Hence, the coefficient of partial derivative of inductance to position in the produced electromagnetic torque (7) is always positive.

For the uncertain system described by (22), the control objective is to track desired speed $\omega$ in the presence of unstructured uncertainties, unknown parameters and external time-varying torque disturbances. In order to develop the control algorithm, the tracking error and two error metric functions are defined as $e=\omega^{*}-\omega, S=e(t)+\dot{e}(t)$ and $S_{r}=\ddot{\omega}(t)+\dot{e}(t)$.

Theorem. Consider the uncertain perturbed nonlinear SRM dynamic model (22). The reference speed tracking, is ensured by using the followed control law:

$$
u=D_{0} S_{r}+\left(\frac{1}{2} \dot{D}_{0}+K\right) S+u_{1}+u_{2}
$$

where:

$$
\begin{gathered}
u_{1}=\frac{1}{2} \hat{\mathrm{z}} S+\hat{\mathrm{z}}^{2} \frac{S S_{r}{ }^{2}}{|S|\left|S_{r}\right| \hat{\mathrm{z}}+\frac{1}{2} \delta e^{-\sigma t}} \\
u_{2}=\hat{\mathrm{w}}^{2} \frac{\Psi \Psi^{T} S}{\left\|\Psi^{T} \mathrm{~S}\right\| \hat{\mathrm{w}}+\frac{1}{2} \delta e^{-\sigma t}}
\end{gathered}
$$

where $K$ is a positive gain and $u_{1}, u_{2}$ are adaptive subcontrollers. The first adaptive subcontroller $u_{1}$ deals with unstructured uncertainties and $u_{2}$ tackles timevarying external disturbances. Exponential parameters $\delta$ and $\sigma$ are some positive constants, selected by the designer. Moreover, $\hat{Z}$ and $\hat{W}$ are updated by the followed adaptation mechanisms:

$$
\begin{gathered}
\dot{\hat{\mathrm{z}}}=\gamma_{\mathrm{z}}\left(|\mathrm{S}|\left|S_{r}\right|+\frac{1}{2} \mathrm{~S}^{2}\right) \\
\dot{\hat{\mathrm{w}}}=\gamma_{\mathrm{w}}\left\|\psi^{T} \mathrm{~S}\right\|
\end{gathered}
$$

where $\gamma_{\mathrm{z}}$ and $\gamma_{\mathrm{w}}$ are the positive adaptation gains.

Proof. Choosing the Lyapunov function as:

$$
V(e, \dot{e}, \mathrm{z}, \mathrm{w})=K e^{2}+\frac{1}{2} D S^{2}+\frac{1}{2 \gamma_{\mathrm{z}}} \tilde{\mathrm{z}}^{2}+\frac{1}{2 \gamma_{\mathrm{w}}} \tilde{\mathrm{w}}^{2}
$$

where $\tilde{\mathrm{Z}}=\mathrm{Z}-\hat{\mathrm{Z}}$ and $\tilde{\mathrm{W}}=\mathrm{W}-\hat{\mathrm{W}}$ denotes the estimation error, the time derivative of $V$ is:

$$
\dot{V}=2 K e \dot{e}+S(D \ddot{e}+D \dot{e})-\frac{1}{\gamma_{\mathrm{z}}} \tilde{\mathrm{z}} \dot{\hat{\mathrm{Z}}}-\frac{1}{\gamma_{\mathrm{w}}} \tilde{\mathrm{w}} \dot{\hat{\mathrm{w}}}
$$


By Eq. (22), the error dynamic may be written as:

$$
D \ddot{e}=D \ddot{\omega}^{*}-u+J^{-1} D \mathrm{~T}_{\mathrm{u}}+\mathbf{C Q}+\frac{1}{2} \dot{D} S
$$

Incorporating the second-order error dynamic (34) and control law (27) in (33) with taking into account assumption 1, (33) can be represented as:

$$
\begin{aligned}
\dot{V} & =2 K e \dot{e}-K S^{2} \\
& +S\left(\Delta D\left(\ddot{\omega}^{*}+\dot{e}\right)+J^{-1} D \mathrm{~T}_{\mathrm{u}}+\mathbf{C Q}+\frac{1}{2} \frac{\mathrm{d} \Delta D}{\mathrm{dt}} S-u_{1}-u_{2}\right) \\
& -\frac{1}{\gamma_{\mathrm{z}}} \tilde{\mathrm{z}} \dot{\hat{\mathrm{z}}}-\frac{1}{\gamma_{\mathrm{w}}} \tilde{\mathrm{w}} \dot{\hat{\mathrm{w}}}
\end{aligned}
$$

By assumption 2 and $3, \dot{V}$ is bounded as:

$$
\begin{aligned}
\dot{V} \leq & -K e^{2}-K \dot{e}^{2}+z\left(|S|\left|S_{r}\right|+\frac{1}{2} S^{2}\right)+w \| \Psi^{T} \mathrm{~S} \mid \\
& -S u_{1}-S u_{2}-\frac{1}{\gamma_{\mathrm{z}}} \tilde{\mathrm{z}} \dot{\hat{\mathrm{Z}}}-\frac{1}{\gamma_{\mathrm{w}}} \tilde{\mathrm{w}} \dot{\hat{\mathrm{w}}}
\end{aligned}
$$

By adaptive subcontrollers (28) and (29), one obtains:

$$
\begin{gathered}
-S u_{1} \leq-\frac{1}{2} \hat{\mathrm{z}} \mathrm{S}^{2}-\hat{\mathrm{z}}|S|\left|S_{r}\right|+\frac{1}{2} \delta e^{-\sigma t} \\
-S u_{2} \leq-\left\|\Psi^{T} \mathrm{~S}\right\| \hat{\mathrm{w}}+\frac{1}{2} \delta e^{-\sigma t}
\end{gathered}
$$

Substituting the inequalities (37) and (38) in (36), (36) can be represented as:

$$
\begin{aligned}
\dot{V} \leq & -K e^{2}-K \dot{e}^{2}+\tilde{\mathrm{z}}\left(|S|\left|S_{r}\right|+\frac{1}{2} S^{2}\right)+\tilde{\mathrm{w}}\left\|\psi^{T} \mathrm{~S}\right\|+\delta e^{-\sigma t} \\
& -\frac{1}{\gamma_{\mathrm{z}}} \tilde{\mathrm{z}} \dot{\hat{\mathrm{z}}}-\frac{1}{\gamma_{\mathrm{w}}} \tilde{\mathrm{w}} \dot{\hat{\mathrm{w}}}
\end{aligned}
$$

Using update laws (30) and (31) in (39) yields:

$$
\dot{V} \leq-K e^{2}-K \dot{e}^{2}+\delta e^{-\sigma t}
$$

By (40) it can be concluded that:

$$
\begin{aligned}
& \dot{V} \leq-K e^{2}+\delta e^{-\sigma t} \\
& \dot{V} \leq-K \dot{e}^{2}+\delta e^{-\sigma t}
\end{aligned}
$$

Now, integrating (41-a) in $0 \leq T<\infty$ results in:

$$
\begin{aligned}
& K \int_{0}^{T}|e(t)|^{2} \mathrm{~d} t+V(e(T), \dot{e}(T), \tilde{z}(T), \tilde{w}(T)) \\
& \leq V(e(0), \dot{e}(0), \tilde{z}(0), \tilde{w}(0))+\frac{\delta}{\sigma}\left(1-e^{-\sigma T}\right)
\end{aligned}
$$

Which implies that $\int_{0}^{T}|e(t)|^{2} \mathrm{~d} t<\infty$, i.e. is square integrable. On the other hand, by (40), one can conclude:

$$
\dot{V} \leq-K|e|^{2}+\delta
$$

For any small (near zero) bound $\varepsilon>0$, the inequality (43) shows that by choosing $K>\delta / \varepsilon^{2}$, there exists a $\kappa>0$ such that when $|e|>\varepsilon$ the time derivative of Lyapunov function becomes negative; i.e.,

$$
\dot{V} \leq-\kappa e^{2} \leq 0
$$

So, the tracking error is bounded. Meanwhile, by using the inequality (41-b), and following the similar procedure it can be concluded that $\dot{e}(t)$ is bounded. Thus, by the Barbalat's lemma, boundedness of all closed-loop signals and the convergence of tracking error $e(t)$ despite the system uncertainties and external torque disturbance is guaranteed.

Remark 3. The exponential terms, formed by $\delta$ and $\sigma$ in (28) and (29) are used to prevent chattering in the control signal. The greater value of such parameters provides the smaller chattering in control signal. On the other hand, there is a trade-off between the smoothness of control effort and the speed of tracking error convergence.

Remark 4. From a theoretical viewpoint, choosing any $K>\delta / \varepsilon^{2}$ ensures holding (44), for any $\varepsilon>0$, even if $K$ is very large. In practice, selecting an arbitrarily large $K$ may be impossible and a certain error bound is allowed in controller design.

Remark 5. In order to alleviate the increase in the estimation values $\hat{Z}$ and $\hat{W}$ without bound occurring in the case of imperfect implementation of adaptation mechanism (30) and (31), an effective modification is adopted here. To this end, substitute the mentioned update laws with:

$$
\begin{gathered}
\dot{\hat{\mathrm{z}}}=\mu_{\mathrm{z}}\left(|\mathrm{S}|\left|S_{r}\right|+\frac{1}{2} \mathrm{~S}^{2}\right) \\
\dot{\hat{\mathrm{w}}}=\mu_{\mathrm{w}}\left\|\psi^{T} \mathrm{~S}\right\|
\end{gathered}
$$

where

$$
\begin{aligned}
& \mu_{\mathrm{z}}= \begin{cases}\gamma_{\mathrm{z}} & \text { if }|e|>v \\
0 & \text { otherwise }\end{cases} \\
& \mu_{\mathrm{w}}= \begin{cases}\gamma_{\mathrm{w}} & \text { if }|e|>v \\
0 & \text { otherwise }\end{cases}
\end{aligned}
$$

Which ensures that all the signals and states of the closed loop system are bounded and $|e|$ is robustly converged to a (small) prescribed bound $v>0$. Briefly discussing, adaptation mechanisms (45) and (46) are activated whenever the norm of the tracking error exceeds 
the prescribed bound $v>0$ and, consequently, the instability due to the increase in $\hat{Z}$ and $\hat{\mathrm{W}}$ is alleviated.

\section{Simulation and Experimental Results}

\subsection{Disturbance rejection and tracking performance study}

Simulation results are presented here for a SRM with the parameters, given in Appendix B. The block diagram of the closed-loop drive control system, using the proposed adaptive control, is shown in Fig. 1. The experimentally measured flux linkage curves of used SRM are shown in Fig 2. In the following, the system performance is evaluated despite the variation of load torque, as an external disturbance, and compared with that of ASMC. To this end, the ASMC parameters are selected as $K_{1}=20$, $\mathrm{c}=8$ and $\rho=0.3$. The proposed adaptive control parameters are $K=10, \gamma_{z}=0.07, \gamma_{w}=0.03$ and exponential terms are $\delta=5$ and $\sigma=1$. Moreover, the turn on and turn off angles is assumed to be constant and equal to $33^{\circ}$ and $56^{\circ}$, respectively. To demonstrate the performance of the SRM drive with the proposed control schemes, the load torque is abruptly increased from 7 to $12 \mathrm{Nm}$ at $0.5 \mathrm{sec}$, as an external disturbance while the reference speed is 1200 rpm. The capability of the proposed adaptive method in external load disturbance rejection, demonstrated in Fig. 3, shows a robustness is achieved, compared with the ASMC. Fig. 4 shows the adaptation procedures of the proposed

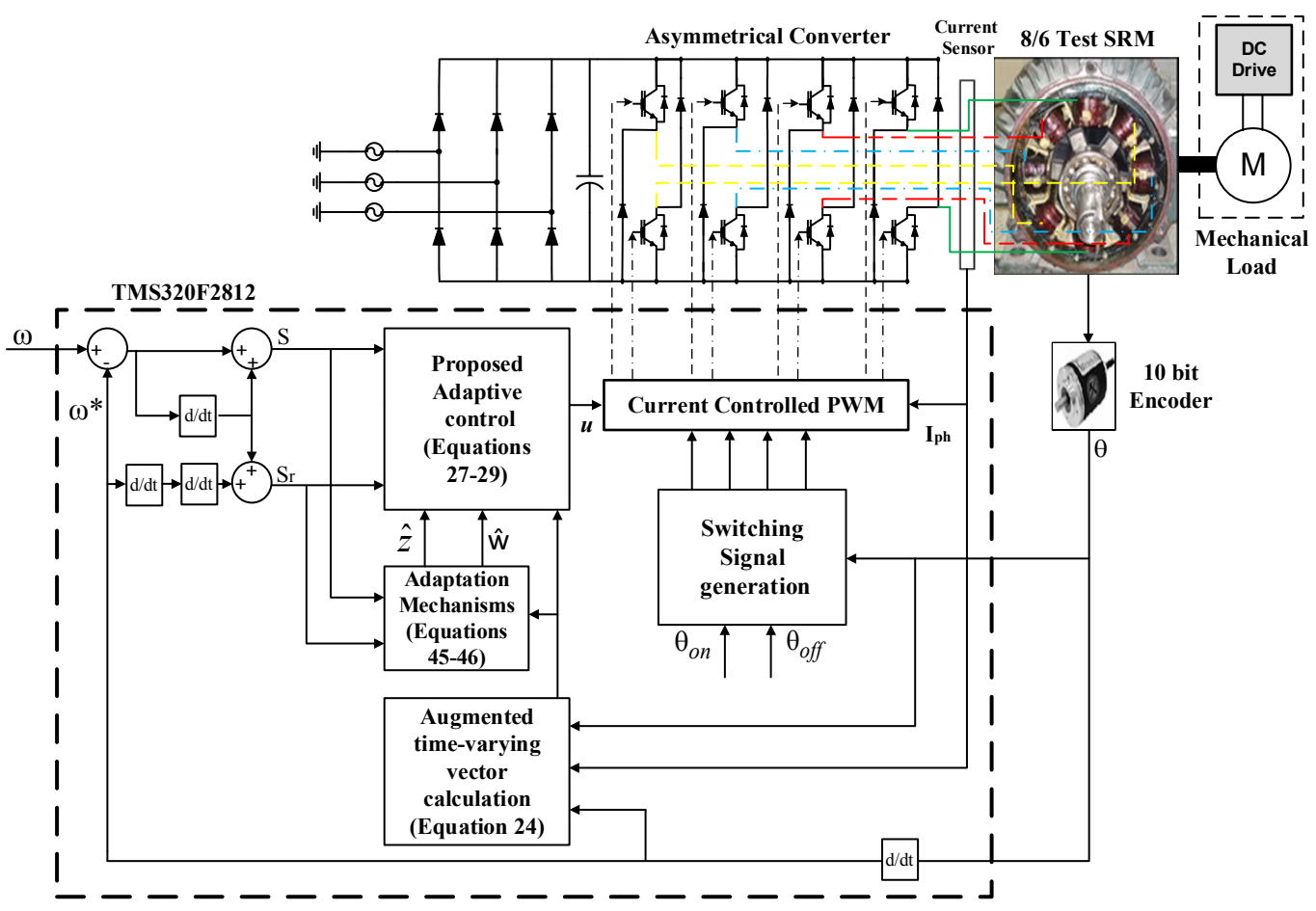

Fig. 1. Proposed adaptive speed control implementation

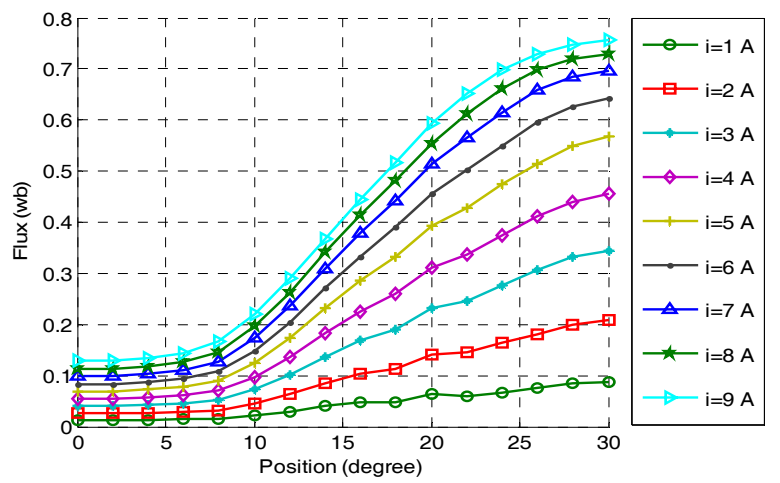

Fig. 2. Flux linkage vs. current vs. rotor position characteristics of prototype $8 / 6 \mathrm{SRM}$

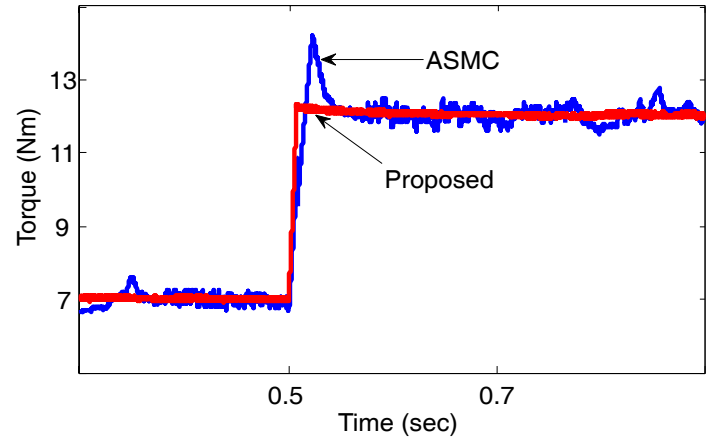

Fig. 3. Performance comparison in load disturbance rejection 


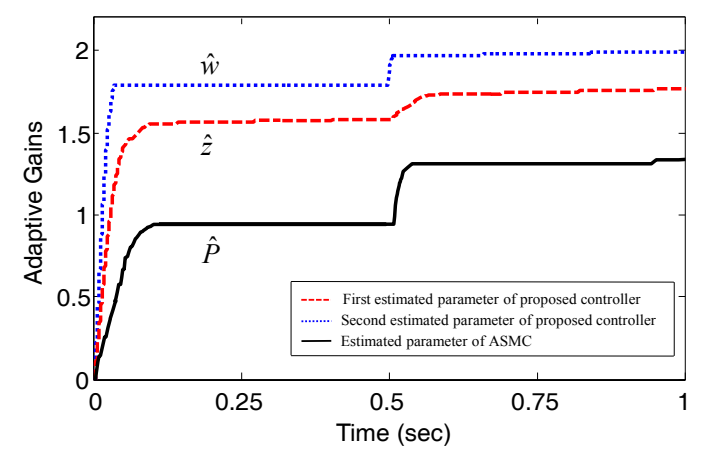

Fig. 4. The adaptive gains of ASMC and proposed adaptive controller

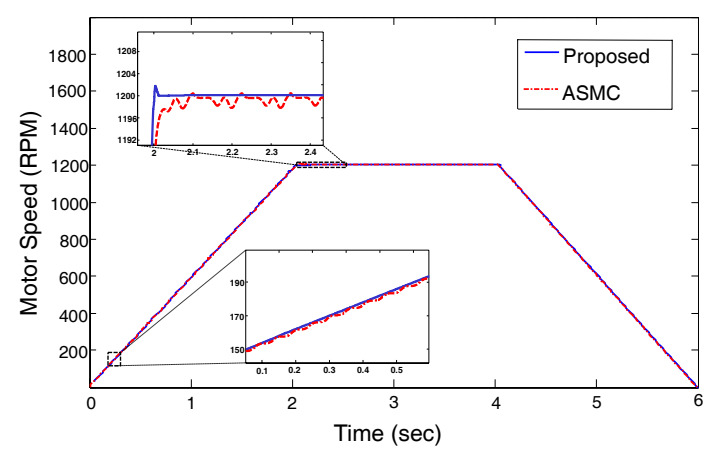

Fig. 5. Four quadrant speed response of SRM

adaptive-based controllers, to overcome such system perturbations. To demonstrate the effectiveness of speed control of the proposed adaptive method a trapezoidal like reference including accelerated and decelerated part is also used to test the controllers. It is shown in Fig. 5 that the speed response with the proposed adaptive controller is more precise than ASMC.

\subsection{Robustness against parametric uncertainty}

To investigate the behavior of proposed control method under parametric variations, the inertia of the motor, the motor load friction, and the stator resistance are let increased by $100 \%$ of the nominal value. Such variations are effectively compensated by the proposed adaptive controller, as illustrated in Fig. 6.

\subsection{Rejection of time-varying load perturbations}

Applying the time-varying load torque with the mean torque values of $12 \mathrm{Nm}$ and $7 \mathrm{Nm}$ is shown in Figs. 7 and 8, respectively. In the first case, the load torque, applied to the motor, is perturbed by a Gaussian noise with variance of $2.5 \mathrm{Nm}$ and in the second case, the perturbation has 1.5 $\mathrm{Nm}$ value. These kinds of load torque perturbations are selected to emulate the electric vehicle driving torque condition [24]. The results show only a small ripple remains in the steady state by applying the proposed
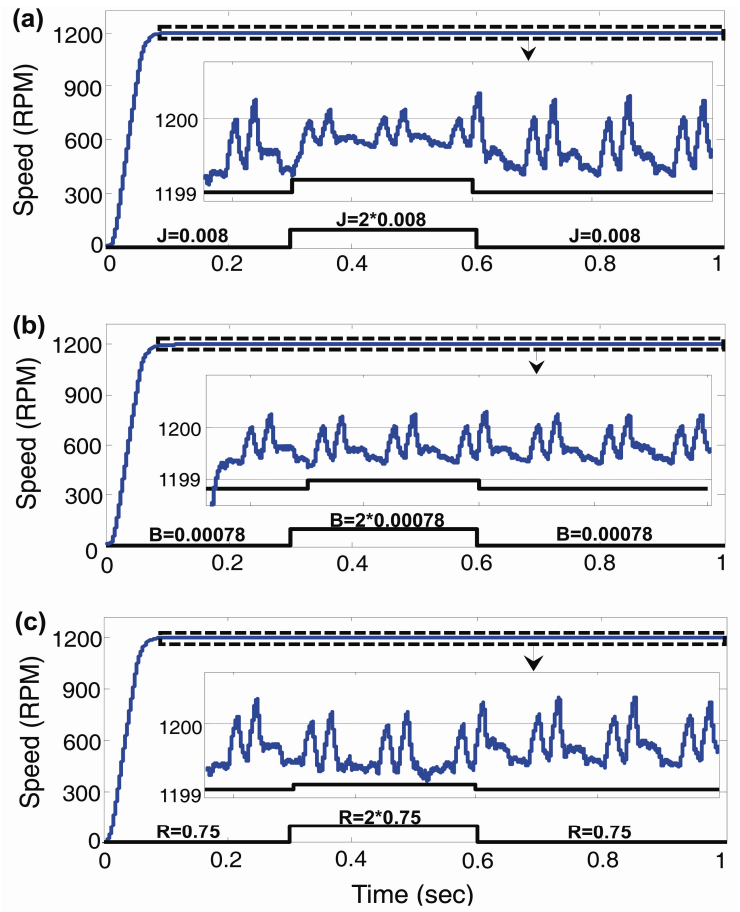

Fig. 6. Speed response despite the $100 \%$ variation in J, B, and $\mathrm{R}$

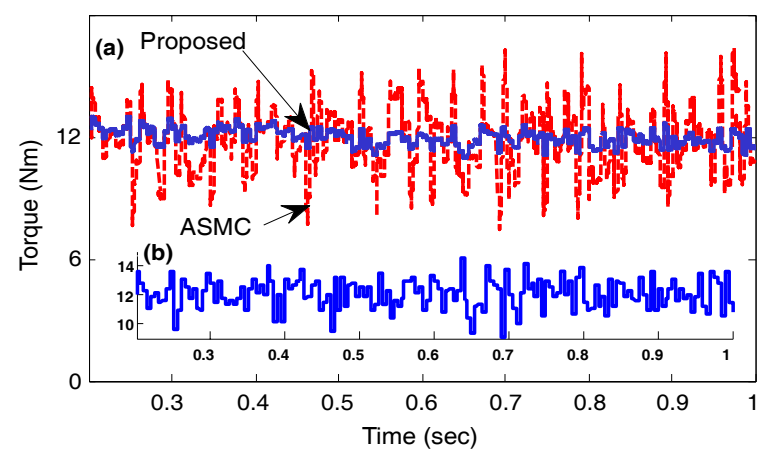

Fig. 7. (a) Motor torque, and (b) applied time-varying load with a mean value of $12 \mathrm{Nm}$ in the presence of a Gaussian perturbation with variance of $2.5 \mathrm{Nm}$

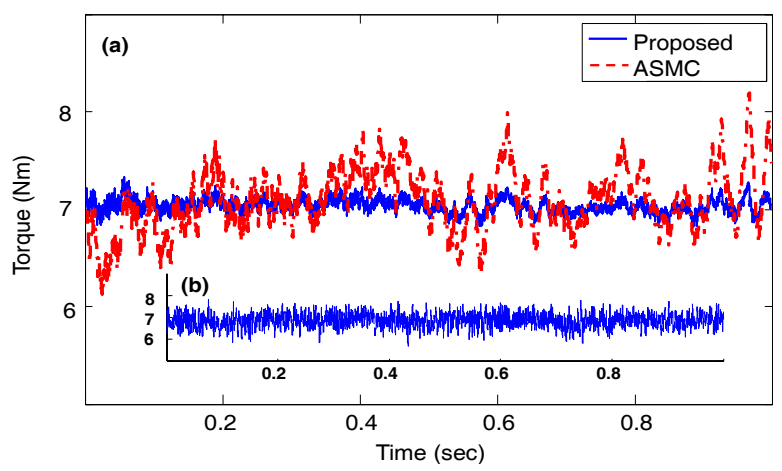

Fig. 8. (a) Motor torque, and (b) applied time-varying load with a mean value of $7 \mathrm{Nm}$ in the presence of a Gaussian perturbation with variance of $1.5 \mathrm{Nm}$ 


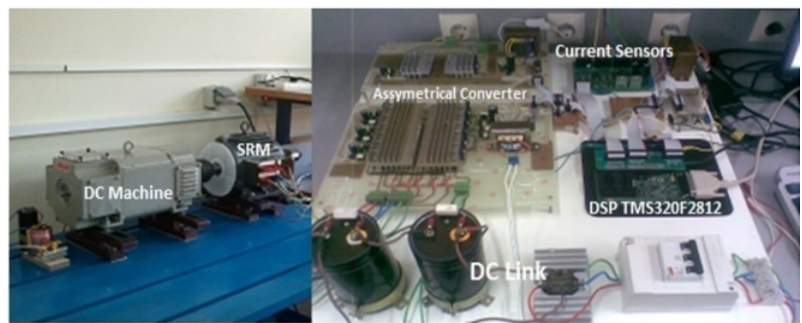

Fig. 9. The laboratory setup for experimental study

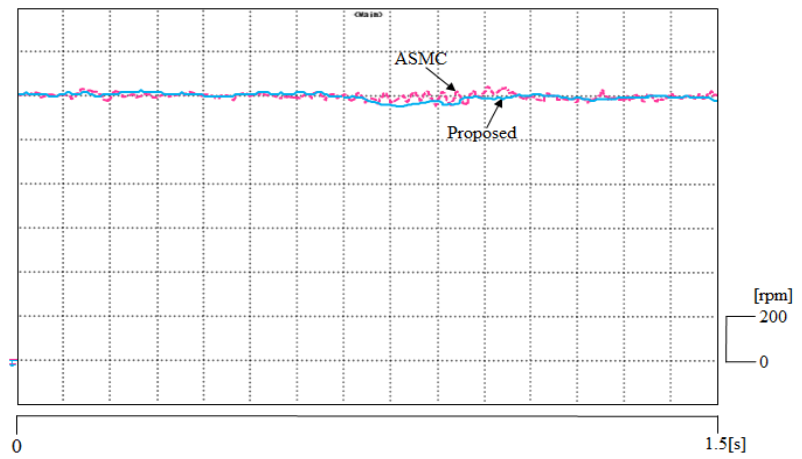

Fig. 10. Speed response performance of the proposed adaptive control in experiment under abrupt load change, compared with that of ASMC

adaptive control scheme.

\subsection{Experimental results}

A SRM drive with same parameters of Appendix B is implemented to experimentally verify the proposed control scheme. The experimental setup is based on a fixed-point TMS320F2812 DSP. This platform includes four dual pulse-width-modulation (PWM) channels (8 channels total), 4 analog-to-digital converters (ADCs), and a speedencoder input. Fig. 9 illustrates the laboratory setup of the DSP-based drive system. The experimental hardware, used for evaluating the 8/6 SRM drive, consists of: (i) The asymmetrical converter, implemented by using IXYS IGBT with a gate driver TLP250 and fast power diodes DSEI 20-12A with a reverse recovery time, less than 40 nanoseconds. (ii) Hall-type galvanic isolation CSNE151104 Honeywell sensors for measuring the phase currents. (iii) A 10-bit absolute encoder Autonics EP50S8, used to determine the rotor position. The results are obtained under the speed loop switching frequency of $10 \mathrm{kHz}$.

Some experimental results are presented here by implementing the proposed and adaptive sliding mode controllers, from a comparative viewpoint. Figs. 10 and 11 demonstrate the speed response, respectively under an abrupt external load change from 7 to $12 \mathrm{Nm}$ at $0.7 \mathrm{sec}$ and under a step change in the reference speed from 600 to 1200 RPM. Torque responses are presented only in simulation due to lack of torque meter equipment. However,

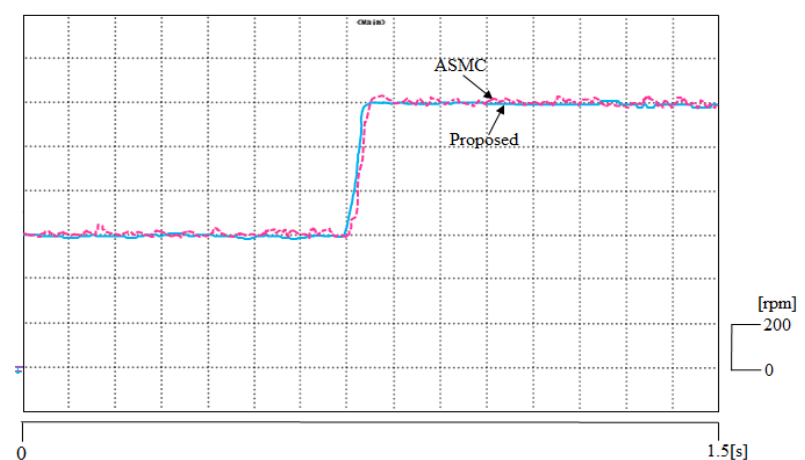

Fig. 11. Speed response in steady state performance of the proposed adaptive control in experiment, under step increment of reference speed, compared with that of ASMC

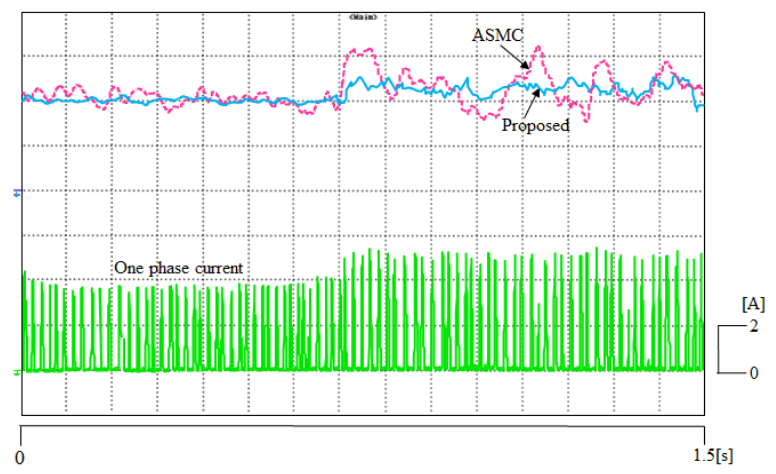

Fig. 12. Control effort and phase current of the proposed adaptive control and ASMC in experiment under abrupt load change

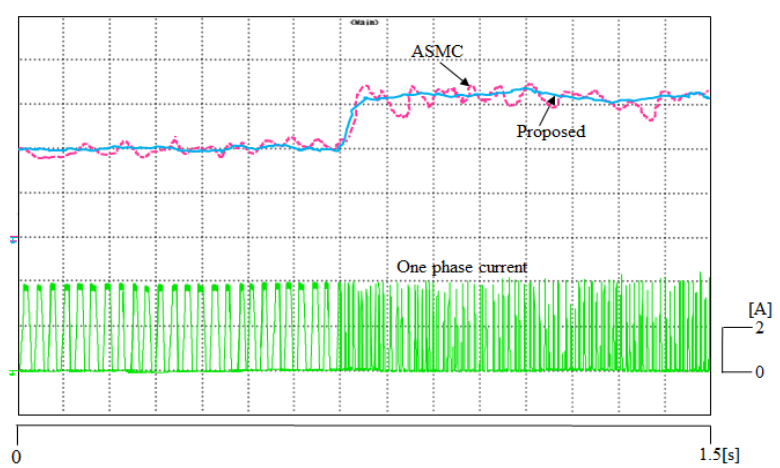

Fig. 13. Control effort and phase current of the proposed adaptive control and ASMC in experiment, under step increment of reference speed

other results such as speed response is shown in both simulation and experiment. The results show that the proposed controller significantly improves the ripple-free performance and successfully eliminates the chattering in the control efforts, as shown in Figs. 12 and 13. By comparing the results of experimental work with those of the simulation study, the proposed controller performance 
is confirmed. The results of implementing the proposed adaptive controller not only confirm the better performance in smoothing the control effort but also demonstrate the time-varying load disturbance attenuation property.

\section{Conclusion}

Two nonlinear adaptive control algorithm are developed for speed control of SRM in the application of EVs, especially in urban areas that the speed is low and the driving torque is highly fluctuated. This condition is emulated by applying a Gaussian noise to perturb the load torque. First, an adaptive sliding mode control (ASMC) is designed to yield a chattering-free control algorithm. However, linearized SRM model is used and only mechanical uncertainty of the dynamic model is considered. To implement a high performance control algorithm for removing these drawbacks, an improved adaptive control, is proposed. An augmented uncertainty is defined taking into account the SRM full nonlinear dynamic model and the system uncertainties as well as time-varying load perturbations. The upper bound of defined augmented uncertainty is not required to be known to make the control gain small enough in order to decrease the chattering and efforts of the controller. Moreover, the exponential terms are used to prevent chattering in the control signal. The proposed adaptive controller is capable of achieving torque ripple minimization with smooth control effort. The simulation and experimental results confirm the robustness against model uncertainties and time-varying load torque.

\section{Acknowledgment}

This work was supported by the Human Resources Program in Energy Technology of the Korea Institute of Energy Technology Evaluation and Planning (KETEP) granted financial resource from the Ministry of Trade, Industry \& Energy, Republic of Korea (No.2016 4010200940)

\section{References}

[1] K. Kiyota, H. Sugimoto, and A. Chiba, "Comparing Electric Motors: An analysis using four standard driving schedules," IEEE Ind. Appl. Mag., Vol. 20, No.4, pp.12-20, Aug. 2014.

[2] K. M. Rahman, B. Fahimi, G. Suresh, A. V. Rajarathnam, and M. Ehsani, "Advantages of switched reluctance motor applications to EV and HEV: design and control issues," IEEE Trans. Ind. Appl., Vol. 36, No. 1, pp. 111-121, 2000.

[3] W. Sun, Y. Li, J. Huang, and N. Zhang, "Vibration effect and control of In-Wheel Switched Reluctance
Motor for electric vehicle," Journal of Sound and Vibration, Vol. 338, pp. 105-120, 2015.

[4] H. C. Chang, and C. M. Liaw, "Development of a Compact Switched-Reluctance Motor Drive for EV Propulsion With Voltage-Boosting and PFC Charging Capabilities," IEEE Trans. Vehic. Technol., Vol. 58, No. 7, pp. 3198-3215, Sep. 2009.

[5] H. Cheng, H. Chen, Z. Yang, W. Huang, "Braking Torque Closed-Loop Control of Switched Reluctance Machines for Electric Vehicles," Journal of Power Electronics, Vol. 15, No. 2, pp. 469-478, 2015.

[6] A. Nishimiya, H. Goto, H. J. Guo, and O. Ichinokura, "Control of SR motor EV by instantaneous torque control using flux based commutation and phase torque distribution technique," Power Electronics and Motion Control Conference, pp. 1163-1167, 2008.

[7] N. Chayopitak, R. Pupadubsin, K. Tungpimolrut, P. Somsiri, P. Jitkreeyan, and S. Kachapornkul, "An Adaptive Low-Ripple Torque Control of Switched Reluctance Motor for Small Electric Vehicle," International Conference on Electrical Machines and Systems, pp. 3327-3332, 2008.

[8] F. U. Syed, M. L. Kuang, and H. Ying, "Active Damping Wheel-Torque Control System to Reduce Driveline Oscillations in a Power-Split Hybrid Electric Vehicle," IEEE Trans. Vehic. Technol., Vol. 58, No. 9, pp. 4769-4785, 2009.

[9] P. Krishnamurthy, W. Lu, F. Khorrami, and A. Keyhani, "Robust force control of an SRM-based electromechanical brake and experimental results," IEEE Trans. Control Syst. Technol. Vol. 17, No. 6, pp. 1306-1317, 2009.

[10] N. Ouddah, M. Boukhnifer, A. Chaibet, and E. Monmasson, "Experimental Robust $\mathrm{H} \infty$ Controller Design of Switched Reluctance Motor for Electrical Vehicle Application," IEEE Conf. Control Application, pp. 1570-1575, 2014.

[11] D. H. Lee, J. Liang, Z. G. Lee, J. W. Ahn, “A simple nonlinear logical torque sharing function for low torque ripple SR drive," IEEE Trans. Ind. Electron., Vol. 56, No. 8, pp. 3021-3028, Aug. 2009.

[12] V. P. Vujicić, "Minimization of torque ripple and copper losses in switched reluctance drive," IEEE Trans. Power Electron., Vol. 27, No. 1, pp. 388-399, 2012.

[13] J. Ye, B. Bilgin, and A. Emadi, "An offline torque sharing function for torque ripple reduction of switched reluctance motor drives," IEEE Trans. Energy Conv., Vol. 30, No. 2, pp. 726-735, 2015.

[14] J. Ye, B. Bilgin, and A. Emadi, "An extended-speed low-ripple torque control of switched reluctance motor drives," IEEE Trans. Power Electron., Vol. 30, No. 3, pp. 1457-1470, 2015.

[15] M. Rafiq, S. Rehman, F. Rehman, Q. R. Butt, and I. A. Awan, "Second order sliding mode control design of a switched reluctance motor using super twisting 
algorithm," Simulat. Model Pract. Theory, Vol. 25, pp. 106-117, 2012.

[16] J. Ye, P. Malysz, and A. Emadi, “A fixed-switchingfrequency integral sliding mode current controller for switched reluctance motor drives," IEEE J. Emerging Selected Topics Power Electron., Vol. 3, No. 2, pp. 381-394, June 2015.

[17] Y. Shtessel, M. Taleb, and F. Plestan, "The novel adaptive sliding mode controller for synchronous reluctance motor speed drive," Automatica, Vol. 48, No. pp. 759-769, 2012.

[18] O. Barambones, and P. Alkorta, "Position control of the induction motor using an adaptive sliding mode controller and observers," IEEE Trans. Ind. Electron., Vol. 61, No. 12 pp. 6556-6565, 2014.

[19] J. Lin, K. W. E. Cheng, Z. Zhang, N. C. Cheung, X. Xue, "Adaptive sliding mode technique-based electromagnetic suspension system with linear switched reluctance actuator," IET Electr. Power Appl., Vol. 9, No. 1, pp. 50-59, March. 2014.

[20] D. Panda, and V. Ramanarayanan, "Mutual coupling and its effect on steady-state performance and position estimation of even and odd number phase switched reluctance motor drive," IEEE Trans. Mag., Vol. 43, No. 8, pp. 3445-3456, 2007.

[21] S. K. Sahoo, S. Dasgupta, S. K. Panda, and J. X. Xu, "A Lyapunov function-based robust direct torque controller for a switched reluctance motor drive system," IEEE Trans. Power Electron., Vol. 27, No. 2, pp. 555-564, 2012.

[22] H. K. Khalil, "Nonlinear Systems," 3rd ed., Prentice Hall, 2002.

[23] T. J. E. Miller, "Electronic Control of Switched Reluctance Machines," New York: Newnes, 2001, Chap.3.

[24] K. Nam, S. Oh, and Y. Hori, "Robust yaw stability control for electric vehicles based on active front steering control through a steer-by-wire system", Int. Journal of Auto. Technol., Vol. 13, No. 7, pp. 11691176, 2012.

\section{Appendix}

Appendix A: Derivation of Eq. (9) in detail.

The derivative of Lyapunov function as defined in (9) is:

$$
\dot{V}_{1}=S_{1} \dot{S}_{1}+\frac{1}{\rho} \tilde{P} \dot{\tilde{P}}
$$

where $\quad \tilde{P}=P-\hat{P} \quad$ and $P=\frac{1}{b}\left(\dot{\omega} \Delta a+\Delta b\left(\dot{T}_{e}-\dot{T}_{L}\right)\right) \quad$ is defined from (5) is assumed to be a constant during the sampling period. Hence, it can be concluded that $\dot{\tilde{P}}=-\dot{\hat{P}}$.
By substituting $\dot{S}_{1}$ from (8) into (A.1), and with adding and subtracting $K_{1} S_{1}^{2}$, the derivative of Lyapunov function can be written as:

$$
\begin{aligned}
\dot{V}_{1}= & S_{1} \dot{S}_{1}-\frac{1}{\rho} \tilde{P} \hat{P}=-K_{1} S_{1}^{2}+K_{1} S_{1}^{2} \\
& +S_{1}\left[-(\mathrm{c}+a) \dot{\omega}-b u+b \dot{T}_{\mathrm{L}}-b(\tilde{P}+\hat{P})\right]-\frac{1}{\rho} \tilde{P} \dot{\hat{P}}
\end{aligned}
$$

Finally it can be simplified as:

$$
\begin{aligned}
& \dot{V}_{1}=-K_{1} S_{1}^{2}+S_{1}\left[-(\mathrm{c}+a) \dot{\omega}-b u+b \dot{T}_{\mathrm{L}}-b \hat{P}+K_{1} S_{1}\right] \\
& -b \tilde{P} S_{1}-\frac{1}{\rho} \tilde{P} \dot{\hat{P}}=S_{1}\left[-(\mathrm{c}+a) \dot{\omega}-b(u+\hat{P})+b \dot{T}_{\mathrm{L}}+K_{1} S_{1}\right] \\
& -K_{1} S_{1}^{2}-\tilde{P}\left(\frac{1}{\rho} \dot{\hat{P}}+b S_{1}\right)
\end{aligned}
$$

\section{Appendix B}

Table 1. SRM specifications

\begin{tabular}{c|c}
\hline Rated power & $4 \mathrm{~kW}$ \\
Rated phase current & $9 \mathrm{~A}$ \\
Rated speed & $1500 \mathrm{rpm}$ \\
Number of pole & $8 / 6$ \\
Phase resistance & $0.75 \Omega$ \\
Inertia (J) & $0.008 \mathrm{~N} . \mathrm{ms}^{2}$ \\
Damping Factor (B) & $0.00078 \mathrm{~N} . \mathrm{ms}$ \\
\hline
\end{tabular}

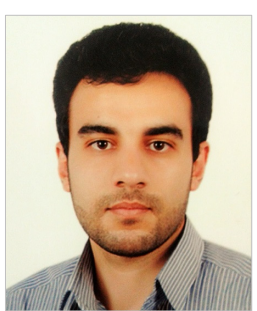

Mohammad Masoud Namazi was born in Isfahan, Iran, in 1986. He received the B.Sc. degree from Shahed University, Tehran, Iran, in 2008 and the M.Sc. degree from Isfahan University of Technology, Isfahan, Iran in 2011, where he is currently working toward the Ph.D. degree in the Department of Electrical and Computer Engineering. $\mathrm{He}$ was a visiting researcher in smart mechatronics lab in Kyungsung University, Busan, Korea from September 2014 to May 2015. His fields of research are control of electric machine drives, automotive applications and control of DC microgrids using renewable energy sources.

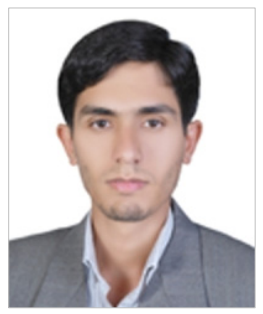

Amir Rashidi was born in Mashhad, Iran, in 1984. He received the B.Sc. degree from Sistan and Baluchestan University, Zahedan, Iran, in 2006 and the M.Sc. degree from Isfahan University of Technology, Isfahan, Iran, in 2009, where he is currently working toward the Ph.D. degree in the 
Department of Electrical and Computer Engineering. His research interests include electric motor drives, power electronics and microprocessor-based control systems.

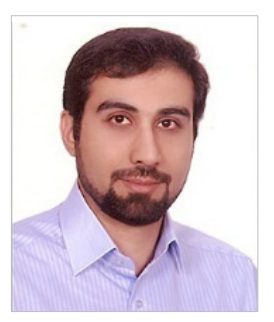

Hamidreza Koofigar He received the M.S. degree in Control Engineering in 2005 and his Ph.D. in Electrical Engineering in 2009, both from Isfahan University of Technology, Iran. He joined the Department of Electrical Engineering, University of Isfahan in 2010, as an assistant professor. His current research interests include robust control, adaptive nonlinear control, and switched systems.

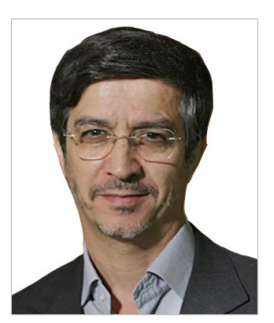

Sayed Morteza Saghaian-Nejad was born in Isfahan, Iran, in 1952. He received his B.S., M.S. and Ph.D. in Electrical Engineering from the University of Kentucky, USA in 1977 , 1979 and 1989, respectively. Since 1979 , he has been a faculty member of Isfahan University of Technology, where he is currently a Professor in electrical and computer engineering department. He is the author or coauthor of various journal and conference papers. His current research interests are in the areas of electrical machines, power electronics and advanced motor drive systems.

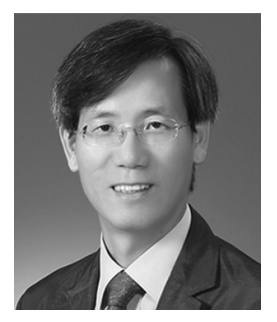

Jin-Woo Ahn was born in Busan, Korea, in 1958. He received the B.S., M.S., and Ph.D. degrees in electrical engineering from Pusan National University, Busan, Korea, in 1984, 1986, and 1992, respectively. He has been with the Department of the Mechatronics Engineering, Kyungsung University, Busan, Korea, as a Professor since 1992. He has been the Director of the Smart Mechatronics Advanced Research and Technology Institute, Kyungsung University, Busan, Korea, since 2008. He has authored five books and more than 130 research papers, and holds more than 30 patents. His research interests include advanced motor drive systems and electric vehicle drives. 